\title{
Dermatology quiz
}

\section{Compiled by Fernando $\mathbf{G} \mathbf{S}^{1}$}

Journal of the Ceylon College of Physicians, 2019, 50, 90-92

1. A 72-year-old woman presented with this non-pruritic, large and thick solitary plaque over forearm of two-years duration. It is scaly with central atrophy. She also noticed slow lateral progression of the lesion along with the central clearance. Black dots were observed over the lesion.

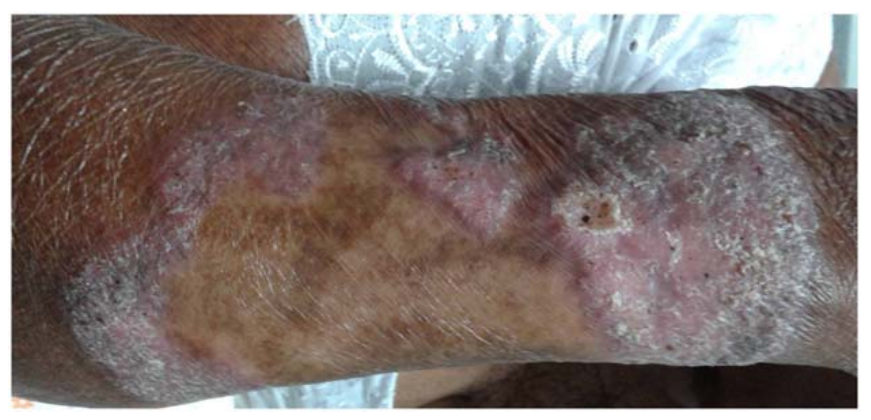

1.1 What is the most likely clinical diagnosis?

1.2 What is the most likely mode of transmission of the disease?

2. An elderly man presented with pruritic facial eruption of five-years duration. His symptoms got worse over time with coarsening of his face.

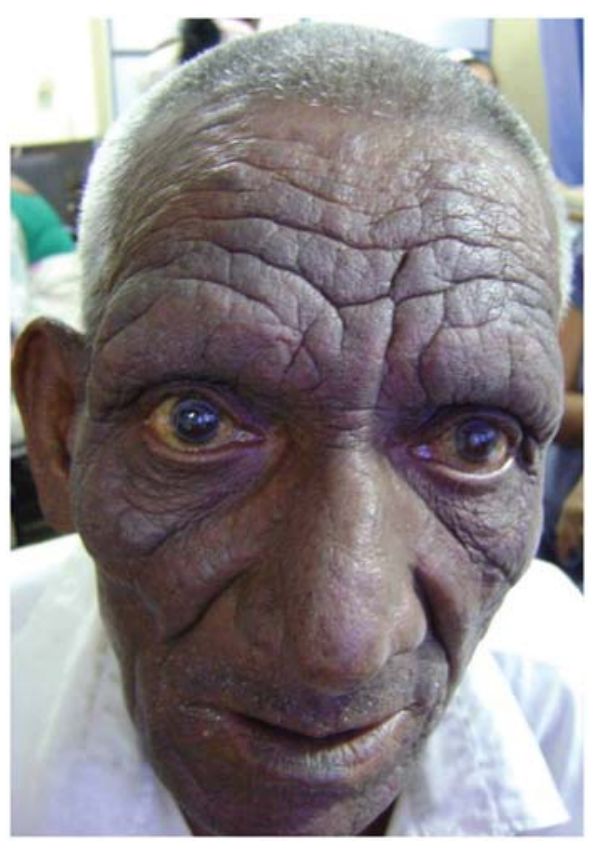

2.1 How would you describe this characteristic facial appearance?

2.2 What is the most likely clinical diagnosis?

\footnotetext{
${ }^{1}$ Consultant Dermatologist (Acting), Base Hospital, Padaviya and Visiting Lecturer, Faculty of Medicine, General Sir John Kotelawala Defence University, Sri Lanka.
}

Correspondence: GSF, e-mail: geehanshan@gmail.com

This is an open-access article distributed under the terms of the Creative Commons Attribution License, which permits unrestricted use, distribution, and reproduction in any medium, provided the original author and source are credited. 
3. A middle-aged man presented with generalized fatigue and myalgia for two weeks. He had facial swelling and an indurated malar eruption which developed abruptly (A). His appearance one week after the commencement of treatment is shown in B.

A

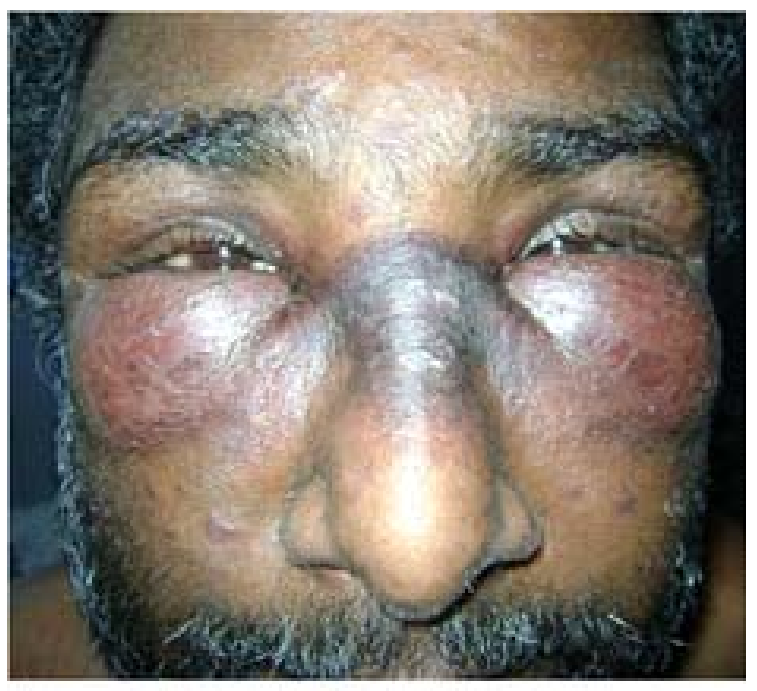

B

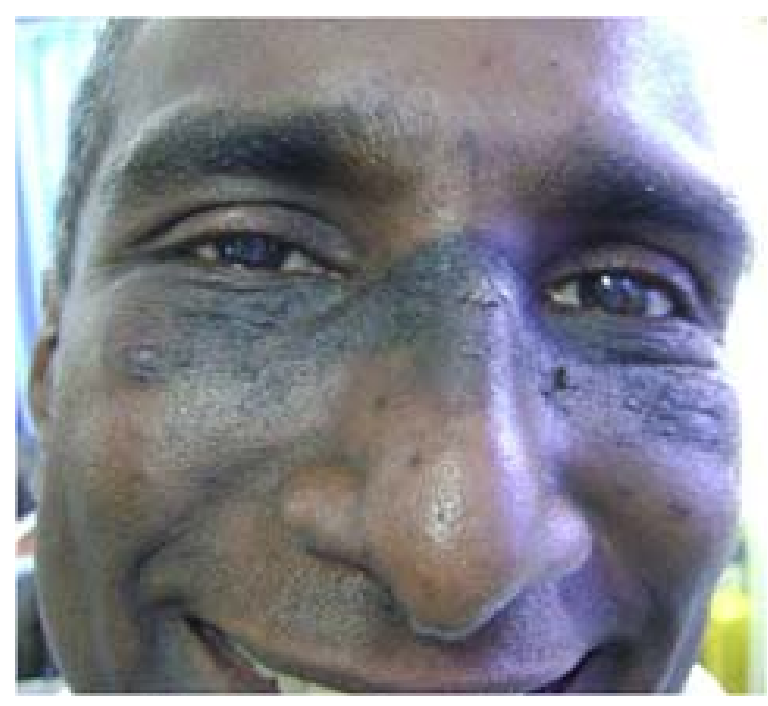

3.1 What is the most likely clinical diagnosis?

3.2 Name one serological test which is specific for this condition?

4. A 15-year-old boy presented with hemorrhagic crusting of lower lip of one-week duration. Upper lip, vermilion border and oral mucosae were spared. Condition was worse in the mornings. He was afebrile and he has not been on any medicine in the recent past.

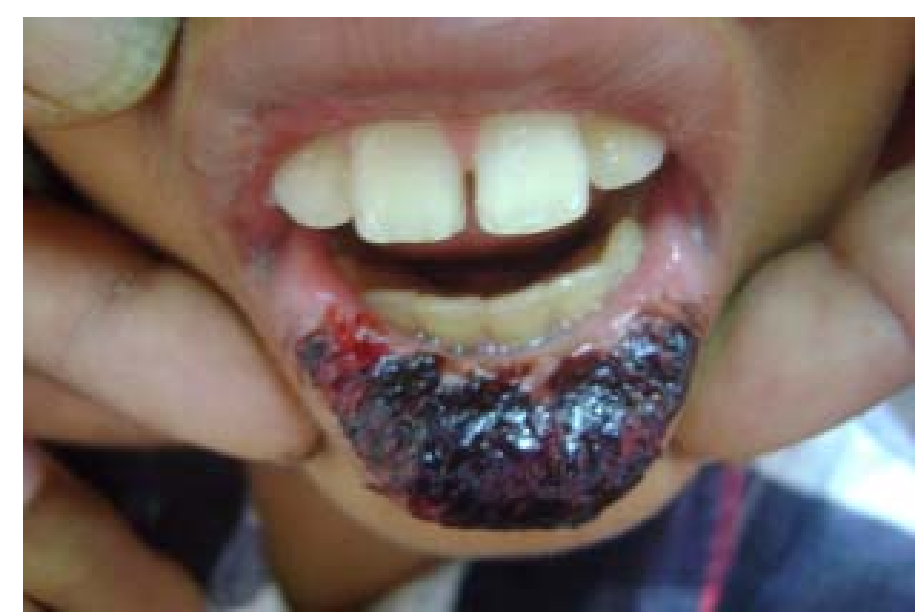

4.1 Describe the characteristic physical sign.

4.2 What is the most likely diagnosis? 
5. A 17-year-old boy presented with this solitary, asymptomatic, verrucous plaque over the left middle finger of six months duration. There was a history of small cut injury one month prior to the onset of the skin lesion. He was an aquarist.

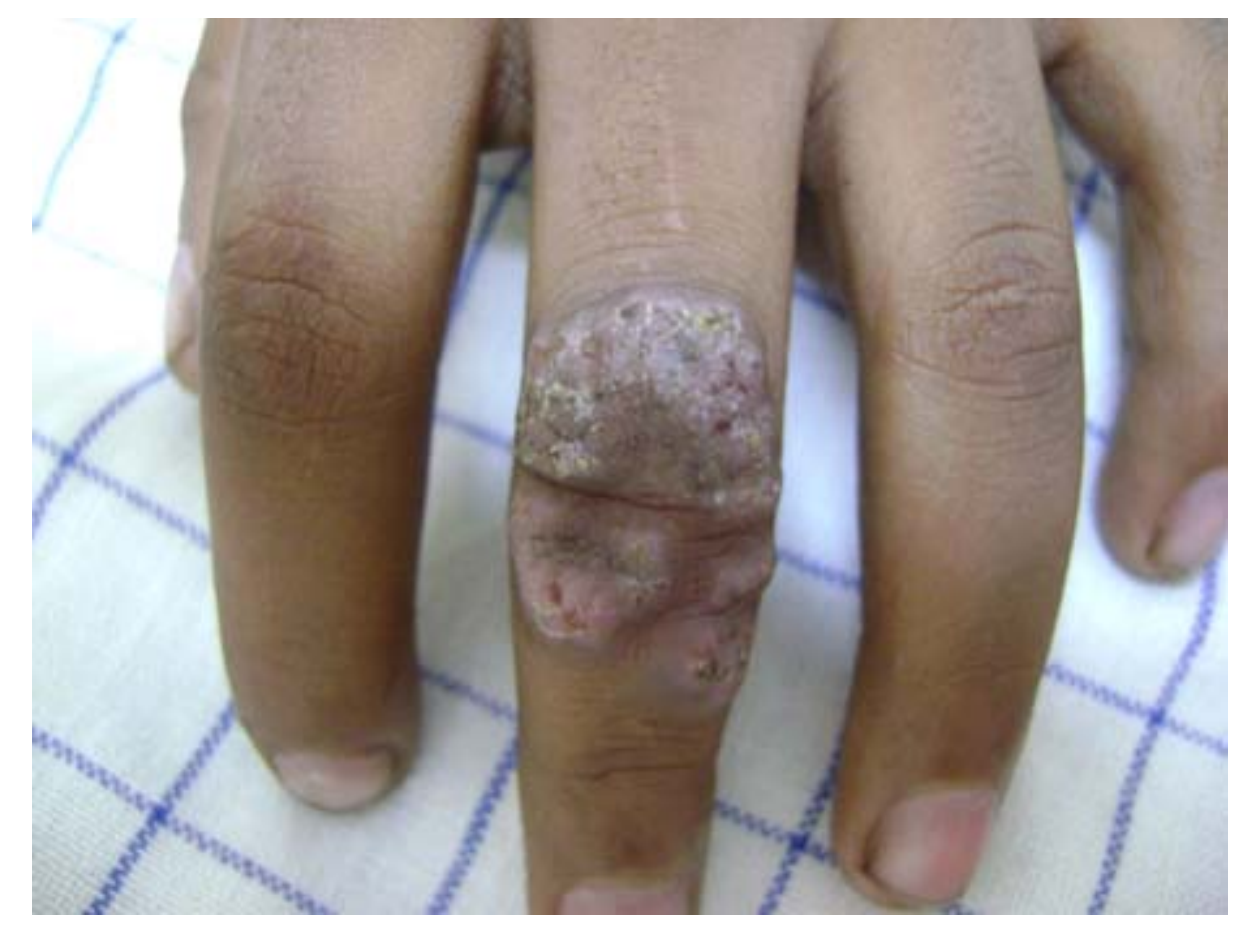

5.1 What is the most likely diagnosis?

5.2 Name three drugs which are indicated as monotherapy or combination therapy.

(Informed consent was obtained from the patients for publication of images)

(Answers on page 94) 